\title{
The Role of Intestinal Fungi and Its Metabolites in Chronic Liver Diseases
}

\author{
Ningning You ${ }^{1}$, Lili Zhuo ${ }^{1}$, Jingxin Zhou ${ }^{1}$, Yu Song ${ }^{2}$, and Junping Shi ${ }^{1}$ \\ ${ }^{1}$ Department of Liver Diseases, The Affiliated Hospital of Hangzhou Normal University, and ${ }^{2}$ Department of Liver Diseases, Zhejiang Chinese \\ Medical University, Hangzhou, China
}

Current studies have confirmed that liver diseases are closely related to intestinal microorganisms; however, those studies have mainly concentrated on bacteria. Although the proportion of intestinal microorganisms accounted for by colonizing fungi is very small, these fungi do have a significant effect on the homeostasis of the intestinal microecosystem. In this paper, the characteristics of intestinal fungi in patients with chronic liver diseases such as alcoholic liver disease, nonalcoholic fatty liver disease and cirrhosis are summarized, and the effects of intestinal fungi and their metabolites are analyzed and discussed. It is important to realize that not only bacteria but also intestinal fungi play important roles in liver diseases. (Gut Liver 2020;14:291-296)

Key Words: Intestinal fungi; Alcoholic liver disease; Cirrhosis; Non-alcoholic fatty liver disease

\section{INTRODUCTION}

The gastrointestinal tracts, as one of the most important and complex micro-ecosystems in human body, harbors large numbers of microorganisms (about $10^{12}$ to $10^{14}$ ), including bacteria, archaea, virus, and fungi; ${ }^{1}$ the enormous quantity and complex structure of intestinal micro-ecology have been a challenge for scientist to have a deep exploration of intestinal microorganism. In recent years, with the advancement of high-throughput sequencing technology, more and more microorganisms have been known and confirmed to be important in maintaining human health, such as immune protection, nutrient absorption, bacterial barrier, anticancer and cancer suppression. ${ }^{2}$ However, there are also potentially pathogenic microorganisms containing in the intestinal micro-ecology causing disruption of intestinal homeostasis and alterations in the intestinal microorganisms which contributes to the pathogenesis of many disorders, including liver disease. Many studies over the past couple of de- cades have documented an important role for intestinal bacteria in liver diseases. Growing evidences indicate that like the bacteria, the intestinal fungi are also closely associated with liver disease.

Intestinal fungi, as an important part of intestinal microecology, though the proportion is very low, its role in human health and disease cannot be ignored. Under physiological conditions, a variety of components on fungal cell wall (including $\beta$-glucan, zymosan, mannan, chitosan, DNA, and RNA) can be recognized by host cells to activate innate and acquired immunity. The reaction inhibits the overgrowth of the intestinal fungi or the colonization of exogenous pathogens. When the host immune system is deficient ${ }^{3}$ or a large number of antibiotics are used, ${ }^{4}$ it will cause changes in the composition of the intestinal microbiota, which will cause a series of liver diseases through the "entero-hepatic axis." In this review, we will combine the current research on intestinal fungus and chronic liver diseases to analyze the relationship between intestinal fungi and alcoholic liver disease (ALD), nonalcoholic fatty liver disease (NAFLD) and liver cirrhosis, try to make people realize that not only bacteria but also intestinal fungi play an important role in liver diseases.

\section{THE GUT MYCOBIOME IN HEALTHY COHORT}

Fungi are detectable in all sections of the gastrointestinal tract of about $70 \%$ of healthy adults, normally at up to 1,000 fungal cells per milliliter or gram of intestinal contents. ${ }^{5} \mathrm{Cul}-$ ture-independent analyses show that fungi constitute less than $0.1 \%$ of the human gut microbiome, ${ }^{6}$ while accounting for only $0.03 \%$ of the fecal microbiota. ${ }^{7}$ Previous traditional culturedependent techniques can only detect a small number of fungi, with the maturity of high-throughput sequencing technology, it has gradually replaced traditional culture techniques as the best method for studying fungi. Reports using next generation se-

Correspondence to: Junping Shi

Department of Liver Diseases, The Affiliated Hospital of Hangzhou Normal University, 126 Wenzhou Road, Hangzhou 310015, China

Tel: +86-57188358060, Fax: +86-57188021730, E-mail: 13957121199@126.com

Received on December 29, 2018. Revised on March 27, 2019. Accepted on April 15, 2019. Published online September 30 , 2019. pISSN 1976-2283 eISSN 2005-1212 https://doi.org/10.5009/gnl18579

(a) This is an Open Access article distributed under the terms of the Creative Commons Attribution Non-Commercial License (http://creativecommons.org/licenses/by-nc/4.0) which permits unrestricted non-commercial use, distribution, and reproduction in any medium, provided the original work is properly cited. 
quencing have found diverse fungal communities in all sections of the human gut, consisting mainly of the phyla Ascomycota, Basidiomycota, Zygomycota, and Chytridiomycota. ${ }^{8-10}$ The predominant commensal fungal species in the human intestine are Candida species, Saccharomyces cerevisiae, and Malassezia species. ${ }^{11}$ The special anatomical and physiological features of the individual compartments of the mouth, stomach and intestine offer disparate ecological niches and they are colonized with site-specific microbe communities. The total number of fungi increases from the ileum to the colon and reaches the highest density in the distal intestine of most monogastric animals. ${ }^{12}$

In 2017, the human microbiome project (HMP) launched the study of the gut mycobiome in human, 317 HMP stool samples were analyzed by sequencing the internal transcribed spacer 2 (ITS2) region as well as the 18S rRNA gene, 701 fungal operational taxonomic units were detected in the sample set, capturing 247 named genera, and the Shannon diversity index varied between 0.004 and 2.94, which was much lower than bacterial communities. The intestinal fungi was dominated by yeast including Saccharomyces, Malassezia, and Candida, and there was the strongest positive correlation between Sarocladium and Fusarium, while Candida and Saccharomyces exhibited the strongest negative correlation. Both inter- and intra-volunteer variability in the HMP cohort were high, revealing that unlike bacterial communities, an individual's mycobiome is no more similar to itself over time than to another person's. Besides, this article also indicates that though 18S rRNA gene and whole-genome shotgun metagenomic sequencing aligned with the results of ITS2 sequencing, ITS2 data provided greater resolution of the mycobiome membership, suggesting that ITS2 sequencing is a more accurate and sensitive method for studying the mycobiome in stool samples. ${ }^{13}$

\section{INTESTINAL FUNGI AND ALCOHOLIC LIVER DISEASE}

Liver acts as a metabolic site for alcohol, when the body excessively consumes alcohol for a long time and exceeds the metabolic load of the liver, it can cause liver damage through multiple routes, and constantly develop into alcoholic fatty liver, alcoholic hepatitis, alcoholic cirrhosis and even liver cancer. ${ }^{14}$ Since 2015 , Szabo ${ }^{15}$ proposed the role of the enterohepatic axis in ALD, the importance of an imbalanced intestinal flora in the pathogenesis of ALD has gradually been recognized. In ALD, increased ethanol and its metabolite acetaldehyde in the intestinal lumen mediate weakening of intestinal tight junctions. Consequently, increased translocation of microbial-associated molecular patterns and gut metabolites, such as acetaldehyde, acetate, elicits intestinal and hepatic inflammatory responses, leading to progressive liver damage. ${ }^{16,17}$

On the basis of a mature mouse model of ALD, Yang et al. ${ }^{18}$ performed ITS sequencing on the feces of mice, and found that the richness and diversity of fungi in the intestinal tract of ALD mice are significantly higher than the control group. Compositional changes in the intestinal mycobiome were characterized by significantly increased proportions of Humicola species, Fusarium, and Aspergillus, while proportions of Candida species decreased. Besides, it is worth noting that the abundance of albicans Candida in ALD group is dramatic overgrowth. The $1,3-\beta$-glucan from fungal cell wall enters the liver by portal vein through the damaged intestinal mucosa, and binds to CLEC7A on the surface of the liver Kupffer cell, then stimulates

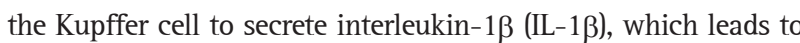
steatosis and necrosis of hepatocyte. Interestingly, overgrowth of Candida was independent of the stage of liver disease, patients with nonprogressive ALD, alcoholic hepatitis, or alcoholic cirrhosis had similar levels of fungal dysbiosis in the intestine. In addition, the study also found that in the ALD patients, the abundance of Epicoccum, unclassified fungi, Galactomyces, and Debaryomyces in the intestinal tract is decreased. Epicoccum fungus is known for its potential to produce diverse classes of biologically active secondary metabolites, and has physiological effects such as anti-oxidation, anticancer, and antibacterial, ${ }^{19,20}$ but the role in ALD has not been studied.

\section{INTESTINAL FUNGI AND NAFLD}

There are few studies on the relationship between intestinal fungi and NAFLD. At present, the most researched is the probiotic Saccharomyces boulardii, which has been widely used in clinical practice. It has the function of neutralizing and degrading bacterial toxins, regulating intestinal flora and immune function. In addition, $S$. boulardii is a beneficial aerobic fungal, which can consume oxygen, produce an anaerobic environment conducive to bifidobacteria and lactic acid bacteria, and inhibit the growth of pathogenic microbiome. ${ }^{21}$

Everard et $a .^{22}$ is the first one who used $S$. boulardii for clinic trial in 2014. After oral administration of $S$. boulardii for 4 weeks, the obese and type 2 diabetic mice has a significant reductions in body weight and fat mass, hepatic steatosis and inflammation are also significantly alleviated. Interestingly, this study found that the effect of $S$. boulardii on host metabolism is related to the local effect of intestinal tract. To a certain extent, it can increase the weight of cecum, and also cause significant changes in the composition of intestinal microorganisms at the level of phylum, family and genus. These changes in intestina microflora may be related to host metabolic response. Liu et al. ${ }^{23}$ performed a similar study on NAFLD mice, which not only got the same conclusions that the mice obtained $S$. boulardii showed a significantly amelioration in hepatic steatosis and inflammation, but also found that $S$. boulardii can regulate the ratio of Escherichia coli/Bacteroides in the intestinal of NAFLD mice, which further illustrates the potential association between gut bacteria and fungi.

In addition, the latest research has also found that metabolic 
syndrome, which is closely related to NAFLD, also shows an imbalance in intestinal fungi. There is a study provides evidence that ingestion of a high-fat diet (HFD) is associated with changes to the fungal, though no difference of richness and diversity was existed between the groups, the abundances of the Alternaria, Saccharomyces, Septoriella, and Tilletiopsis genera; S. cerevisiae; and Tilletiopsis washingtonensis were higher in mice fed standard chow. ${ }^{24}$ Beside, Mar Rodríguez et al. ${ }^{10}$ performed ITS analysis on the fecal microbiota of 27 obese patients and 12 healthy controls to analyze the abundance and diversity of intestinal fungal, though there was no evident difference in fungal abundance between the groups, the obese mice has a significantly lower diversity than the control group. And the abundance of the genus Mucor and Penicillium, which belongs to Zygomycota, was significantly reduced in the nonobese group.

Overall, evidence for a causal role of intestinal fungi in NAFLD in humans is limited because the gold-standard method for assessing and diagnosing NAFLD is still a liver biopsy. Improved noninvasive techniques will help enable well-powered studies to be performed to elucidate the role of the gut mycobiota in NAFLD.

\section{INTESTINAL FUNGI AND END-STAGE LIVER DISEASE}

Cirrhosis is a result of advanced liver diseases such as alcoholism, viral hepatitis, and fatty liver disease. It is characterized by replacement of live tissue by fibrosis and regenerative nodules; these changes lead to loss of liver function. ${ }^{25}$

In recent years, more and more studies have found that intestinal fungi have a close correlation with cirrhosis. Patients with cirrhosis are often accompanied by disorders of the intestinal fungi, the main manifestations were the decrease of fungal diversity and species abundance. The abundance of basidiomycetes in the intestinal tract of patients with cirrhosis is significantly reduced, while Ascomycota has an absolute advantage. For Ascomycota, there is a literature report that it is positively correlated with the incidence of end-stage liver disease, and can predict the short-term hospitalization rate of patients with advanced cirrhosis. ${ }^{26}$ Most of the patients are hospitalized for infection, such as fungal peritonitis, fungemia, fungal esophagitis, etc., most of the pathogens of these infections are Ascomycetes and have been confirmed as intestinal origin. ${ }^{11,27,28}$ This result tells us that liver cirrhosis patients are accompanied by intestinal fungal flora disorder and mucosal permeability destruction at the same time, intestinal fungi can enter the liver or even the whole body through the damaged intestinal mucosa, however, whether the disturbed fungi can in turn aggravate liver damage through the entero-hepatic axis has not been studied.

In another study by Chen et al. ${ }^{29}$ on the correlation between gastrointestinal fungi and varying degrees of hepatitis B virus (HBV) infection, it is suggested that the hepatitis B cirrhosis and the chronic hepatitis B showed higher fungal diversity than the
HBV carriers and the healthy control, and the hepatitis B cirrhosis had the highest diversity and species composition, indicating that the diversity of fungi in the gut is positively correlated with the degree of progression of HBV infection. Besides, the abundance of fungi in hepatitis B cirrhosis was also significantly higher than other groups, this result is consistent with the conclusion that Brown et $a l^{30}$ and Knoke ${ }^{31}$ have confirmed in the preceding years that the increase in the number of fungi was significantly associated with the severity of the disease in HBVinfected patients. The association between chronic hepatitis $B$ cirrhosis and intestinal fungi is related to defects in the host immune response. Mannose-binding protein, a pattern-recognition receptor that binds to mannose on the fungal cell wall and triggers a host immune response, thus playing a key role in combating fungal pathogens. In the course of HBV infection, the lack or dysfunction of mannose-binding proteins may lead to a weakened defense against fungi, leading to increased colonization of these pathogens.

From the above research, we found that liver cirrhosis caused by different etiology showed different changes in the structure and composition of intestinal fungi, which indicates that it is not the state of cirrhosis which causes the changes of intestina fungi, but the intestinal fungi under the action of different etiology that lead to the occurrence of liver cirrhosis.

\section{FUNGAL METABOLITES ON CHRONIC LIVER DISEASE}

Similar to the intestinal fungi, the study on intestinal fungal metabolites are also at a very early stage. We try to elucidate the mechanism of action of these metabolites, combined with the pathogenesis of chronic liver disease, to explore the relationship between them.

\section{Penstyrylpyrone}

As one of the metabolites of Penicillium, penstyrylpyrone can inhibit the activity of protein tyrosine phosphatase 1B (PTP1B), while PTP1B is a major non-transmembrane phosphotyrosine phosphatase, as a negative regulator of the insulin-stimulated signal transduction pathway, ${ }^{32}$ is associated with type 2 diabetes and obesity, may also provide a new target for the treatment of NAFLD.

\section{S. boulardii anti-inflammatory factor}

S. boulardii anti-inflammatory factor, a low molecular weight, water soluble factor, play an important role in S. boulardii antiinflammatory properties and assist to modulate a signal transduction pathway including nuclear factor kappa B (NF- $\mathrm{B}) .^{33}$ While the activation of the NF- $\kappa \mathrm{B}$ pathway can stimulate the release of proinflammatory cytokines such as tumor necrosis factor- $\alpha$, IL-1 $\beta$, and IL- 6 , further promotes the generation of reactive oxygen species and exacerbates liver damage both in ALD and NAFLD. ${ }^{34,35}$ Besides, $S$. boulardii anti-inflammatory 
factor has been suggested to preserve the integrity of tight junctions between enterocytes ${ }^{36}$ and has also been found to increase butyrate concentration (probably due to cooperation with bacteria). ${ }^{37}$ Moreover, butyrate has been proved to exert protective effects against nonalcoholic steatohepatitis development mainly in alleviation of hepatic injury, fibrosis progression, inflammation, and lipid metabolism and intestinal barrier dysfunction. ${ }^{38}$ Above all, $S$. boulardii anti-inflammatory factor may be closely related to chronic liver disease, the direct relationship between them needs to be further studied.

\section{Polypeptides and other S. boulardit-derived molecules}

Polyamines (spermine, spermidine, putrescine), produced by $S$. boulardii, has been found to have a trophic effect on the maturation of enterocytes. Besides, 54-kDa serine protease and 63$\mathrm{kDa}$ phosphatase, produced by $S$. boulardii can directly destroys Clostridium difficile toxin A and degrades endotoxin of pathogenic E. coli respectively. ${ }^{39,40}$ The role of polyamines in chronic liver disease has not been studied.

\section{Farnesol}

Farnesol is a non-sterol isoprenoid that is produced endogenously through the dephosphorylation of farnesyl pyrophosphate, a key branch-point intermediate of the cholesterol biosynthetic pathway. A lot of research has been done on farnesol, in addition to being functional in stimulating mitochondrial generation of ROS and apoptosis ${ }^{41-45}$ as well as decreases intracellular cyclic adenosine monophosphate levels, ${ }^{46,47}$ recent study found isoprenoid farnesol can also modulate lipid metabolism and reduces hepatic triglyceride content in steatotic HepaRG cells. This effect involves activation of at least two nuclear receptors, peroxisome proliferator-activated receptor alpha and farnesoid X receptor. ${ }^{48}$

\section{Altenusin}

Altenusin, a natural nonsteroidal fungal metabolite isolated from the endophytic fungus Alternaria sp., as a novel selective agonist of farnesoid X receptor (FXR), protected mice from HFD-induced obesity by reducing the body weight and fat mass. Administration of Altenusin also decreased the level of blood glucose and serum insulin level, even reversed HFD-induced hepatic lipid droplet accumulation and macrovesicular steatosis. Mechanistically, the metabolic benefits of Altenusin might have been accounted for by the increased insulin sensitivity and suppression of genes that are involved in hepatic gluconeogenesis and lipogenesis. In summary, Altenusin can be regarded as a new class of nonsteroidal FXR agonist that shows promise in treating NAFLD and the associated metabolic syndrome. ${ }^{49}$

\section{FUTURE DIRECTION}

The role of intestinal microorganisms in the occurrence and development of various diseases has been paid more attention currently, and the relationship between intestinal fungi and bacterial has gradually deepened. In a variety of liver diseases, the intestinal fungi shows a certain structural and compositiona changes, which may provide new directions and targets for the treatment of chronic liver disease, such as apply beneficial fung for the treatment of some disease by means of fecal microbiota transplantation. Furthermore, with the increasing incidence of NAFLD and the risk of NAFLD transitioning to steatohepatitis, cirrhosis and liver cancer, there is no effective drug for the treatment of NAFLD, we need to further explore the characteristics of intestinal fungi in patients with NAFLD, to verify the causal effect between NAFLD and fungal dysbiosis, providing a new direction for the treatment of NAFLD.

\section{CONFLICTS OF INTEREST}

No potential conflict of interest relevant to this article was reported.

\section{ACKNOWLEDGEMENTS}

The authors thank all patients who participated in the study, the Science and Technology Planning Project of Hangzhou City (20172016A02), which plays an important role in this article.

\section{ORCID}

Ningning You

Lili Zhuo

Jingxin Zhou

Yu Song

Junping Shi https://orcid.org/0000-0002-1040-4944

https://orcid.org/0000-0002-5626-9581

https://orcid.org/0000-0003-3734-9778

https://orcid.org/0000-0002-1023-4842

https://orcid.org/0000-0001-9434-897X

\section{REFERENCES}

1. Sekirov I, Russell SL, Antunes LC, Finlay BB. Gut microbiota in health and disease. Physiol Rev 2010;90:859-904.

2. Carter BA, Karpen SJ. Intestinal failure-associated liver disease: management and treatment strategies past, present, and future. Semin Liver Dis 2007;27:251-258.

3. Romani L. Immunity to fungal infections. Nat Rev Immunol 2011;11:275-288.

4. Dollive S, Chen YY, Grunberg S, et al. Fungi of the murine gut: episodic variation and proliferation during antibiotic treatment PLoS One 2013;8:e71806.

5. Schulze J, Sonnenborn U. Yeasts in the gut: from commensals to infectious agents. Dtsch Arztebl Int 2009;106:837-842.

6. Qin J, Li R, Raes J, et al. A human gut microbial gene catalogue established by metagenomic sequencing. Nature 2010;464:59-65.

7. Ott SJ, Kuhbacher T, Musfeldt M, et al. Fungi and inflammatory bowel diseases: alterations of composition and diversity. Scand J 
Gastroenterol 2008;43:831-841.

8. Hoffmann C, Dollive S, Grunberg S, et al. Archaea and fungi of the human gut microbiome: correlations with diet and bacterial residents. PLoS One 2013;8:e66019.

9. Suhr MJ, Hallen-Adams HE. The human gut mycobiome: pitfalls and potentials: a mycologist's perspective. Mycologia 2015;107:1057-1073.

10. Mar Rodríguez M, Pérez D, Javier Chaves F, et al. Obesity changes the human gut mycobiome. Sci Rep 2015;5:14600.

11. Wang ZK, Yang YS, Stefka AT, Sun G, Peng LH. Review article: fungal microbiota and digestive diseases. Aliment Pharmacol Ther 2014;39:751-766.

12. Scupham AJ, Presley LL, Wei B, et al. Abundant and diverse fungal microbiota in the murine intestine. Appl Environ Microbiol 2006;72:793-801.

13. Nash AK, Auchtung TA, Wong MC, et al. The gut mycobiome of the Human Microbiome Project healthy cohort. Microbiome 2017;5:153.

14. Voican CS, Perlemuter G, Naveau S. Mechanisms of the inflammatory reaction implicated in alcoholic hepatitis: 2011 update. Clin Res Hepatol Gastroenterol 2011;35:465-474.

15. Szabo G. Gut-liver axis in alcoholic liver disease. Gastroenterology 2015;148:30-36.

16. Schnabl B, Brenner DA. Interactions between the intestinal microbiome and liver diseases. Gastroenterology 2014;146:1513-1524.

17. Chen P, Stärkel P, Turner JR, Ho SB, Schnabl B. Dysbiosis-induced intestinal inflammation activates tumor necrosis factor receptor I and mediates alcoholic liver disease in mice. Hepatology 2015;61:883-894.

18. Yang AM, Inamine $\mathrm{T}$, Hochrath $\mathrm{K}$, et al. Intestinal fungi contribute to development of alcoholic liver disease. J Clin Invest 2017;127:2829-2841.

19. Fatima N, Ismail T, Muhammad SA, et al. Epicoccum SP., an emerging source of unique bioactive metabolites. Acta Pol Pharm 2016;73:13-21.

20. Braga RM, Padilla G, Araújo WL. The biotechnological potential of Epicoccum spp.: diversity of secondary metabolites. Crit Rev Microbiol 2018;44:759-778.

21. Kelesidis T, Pothoulakis C. Efficacy and safety of the probiotic Saccharomyces boulardii for the prevention and therapy of gastrointestinal disorders. Therap Adv Gastroenterol 2012;5:111-125.

22. Everard A, Matamoros S, Geurts L, Delzenne NM, Cani PD. Saccharomyces boulardii administration changes gut microbiota and reduces hepatic steatosis, low-grade inflammation, and fat mass in obese and type 2 diabetic $\mathrm{db} / \mathrm{db}$ mice. MBio 2014;5:e01011e01014.

23. Liu YT, Li YQ, Wang YZ. Protective effect of Saccharomyces boulardii against intestinal mucosal barrier injury in rats with nonalcoholic fatty liver disease. Zhonghua Gan Zang Bing Za Zhi 2016;24:921-926.

24. Heisel T, Montassier E, Johnson A, et al. High-fat diet changes fungal microbiomes and interkingdom relationships in the murine gut. mSphere 2017;2:e00351-17.

25. El Bialy SA, El Kader KF, El-Ashmawy MB. Current progress in antifibrotics. Curr Med Chem 2011;18:3082-3092.

26. Bajaj JS, Liu EJ, Kheradman R, et al. Fungal dysbiosis in cirrhosis. Gut 2018;67:1146-1154.

27. Bajaj JS, O’Leary JG, Reddy KR, et al. Second infections independently increase mortality in hospitalized patients with cirrhosis: the North American consortium for the study of end-stage liver disease (NACSELD) experience. Hepatology 2012;56:2328-2335.

28. Lahmer T, Messer M, Mayr U, et al. Fungal "colonisation" is associated with increased mortality in medical intensive care unit patients with liver cirrhosis. Mycopathologia 2015;179:63-71.

29. Chen Y, Chen Z, Guo R, et al. Correlation between gastrointestinal fungi and varying degrees of chronic hepatitis $\mathrm{B}$ virus infection. Diagn Microbiol Infect Dis 2011;70:492-498.

30. Brown KS, Ryder SD, Irving WL, Sim RB, Hickling TP. Mannan binding lectin and viral hepatitis. Immunol Lett 2007;108:34-44.

31. Knoke M. Gastrointestinal microecology of humans and Candida. Mycoses 1999;42 Suppl 1:30-34.

32. Lee DS, Jang JH, Ko W, et al. PTP1B inhibitory and anti-inflammatory effects of secondary metabolites isolated from the marinederived fungus Penicillium sp. JF-55. Mar Drugs 2013;11:14091426.

33. Sougioultzis S, Simeonidis S, Bhaskar KR, et al. Saccharomyces boulardii produces a soluble anti-inflammatory factor that inhibits NF-kappaB-mediated IL-8 gene expression. Biochem Biophys Res Commun 2006;343:69-76.

34. Okiyama W, Tanaka N, Nakajima T, et al. Polyenephosphatidylcholine prevents alcoholic liver disease in PPARalpha-null mice through attenuation of increases in oxidative stress. J Hepatol 2009;50:1236-1246.

35. Roth CL, Elfers CT, Figlewicz DP, et al. Vitamin D deficiency in obese rats exacerbates nonalcoholic fatty liver disease and increases hepatic resistin and Toll-like receptor activation. Hepatology 2012;55:1103-1111.

36. Czerucka D, Dahan S, Mograbi B, Rossi B, Rampal P. Saccharomyces boulardii preserves the barrier function and modulates the signal transduction pathway induced in enteropathogenic Escherichia coli-infected T84 cells. Infect Immun 2000;68:5998-6004.

37. Schneider SM, Girard-Pipau F, Filippi J, et al. Effects of Saccharomyces boulardii on fecal short-chain fatty acids and microflora in patients on long-term total enteral nutrition. World J Gastroenterol 2005;11:6165-6169.

38. Ye J, Lv L, Wu W, et al. Butyrate protects mice against methionine-choline-deficient diet-induced non-alcoholic steatohepatitis by improving gut barrier function, attenuating inflammation and reducing endotoxin levels. Front Microbiol 2018;9:1967.

39. Castagliuolo I, LaMont JT, Nikulasson ST, Pothoulakis C. Saccharomyces boulardii protease inhibits Clostridium difficile toxin A effects in the rat ileum. Infect Immun 1996;64:5225-5232.

40. Buts JP, Dekeyser N, Stilmant C, Delem E, Smets F, Sokal E. Saccharomyces boulardii produces in rat small intestine a novel 
protein phosphatase that inhibits Escherichia coli endotoxin by dephosphorylation. Pediatr Res 2006;60:24-29.

41. Joo JH, Jetten AM. Molecular mechanisms involved in farnesolinduced apoptosis. Cancer Lett 2010;287:123-135.

42. Rossignol T, Logue ME, Reynolds K, Grenon M, Lowndes NF, Butler G. Transcriptional response of Candida parapsilosis following exposure to farnesol. Antimicrob Agents Chemother 2007;51:2304-2312.

43. Liu P, Luo L, Guo J, et al. Farnesol induces apoptosis and oxidative stress in the fungal pathogen Penicillium expansum. Mycologia 2010;102:311-318.

44. Machida K, Tanaka T. Farnesol-induced generation of reactive oxygen species dependent on mitochondrial transmembrane potential hyperpolarization mediated by $\mathrm{F}(0) \mathrm{F}(1)-\mathrm{ATP} a \mathrm{se}$ in yeast. FEBS Lett 1999;462:108-112.

45. Machida K, Tanaka T, Fujita K, Taniguchi M. Farnesol-induced generation of reactive oxygen species via indirect inhibition of the mitochondrial electron transport chain in the yeast Saccharomyces cerevisiae. J Bacteriol 1998;180:4460-4465.

46. Davis-Hanna A, Piispanen AE, Stateva LI, Hogan DA. Farnesol and dodecanol effects on the Candida albicans Ras1-cAMP signalling pathway and the regulation of morphogenesis. Mol Microbiol 2008;67:47-62.

47. Lorek J, Pöggeler S, Weide MR, Breves R, Bockmühl DP. Influence of farnesol on the morphogenesis of Aspergillus niger. J Basic Microbiol 2008;48:99-103.

48. Pant A, Rondini EA, Kocarek TA. Farnesol induces fatty acid oxidation and decreases triglyceride accumulation in steatotic HepaRG cells. Toxicol Appl Pharmacol 2019;365:61-70.

49. Zheng Z, Zhao Z, Li S, et al. Altenusin, a nonsteroidal microbial metabolite, attenuates nonalcoholic fatty liver disease by activating the farnesoid X receptor. Mol Pharmacol 2017;92:425-436. 